\title{
CULTURA Y SALUD MENTAL. INFLUENCIA DEL MEDIO INSTITUCIONAL EN LOS ENFERMOS PROFUNDOS ADULTOS
}

\author{
FRANCISCO J. MARTINEZ CARRASCO \\ MARIA DOLORES VARGAS LLOVERA \\ Divisón de Antropología \\ Escuela Universitaria de Trabajo Social \\ Universidad de Alicante
}

\section{PLANTEAMIENTO GENERAL DEL PROBLEMA}

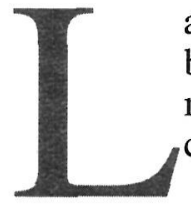

a reacción social ante los que seguían una conducta y hábitos muy alejados de lo considerado como normal, se ha manifestado a lo largo de la historia con unas características muy comunes.

La reclusión en instituciones, el desplazamiento forzado a lugares controlados y lejos de los medios sociales, incluso el aniquilamiento de los enfermos mentales, conforman la historia con que los considerados dentro de la normalidad han respondido, en muchos casos, ante las desviaciones mentales.

La valoración social del desequilibrio psíquico ha sufrido, al igual que la gran mayoría de concepciones sociales y culturales, un proceso de transformación. Aquella persona a que se le reconoce una incapacidad de obra y razón, en la mayoría de las culturas es privada de una existencia equiparable a la de sus semejantes en posesión de las plenas facultades.

En la manera de realizar esta separación y deportación del diferente, influye decisivamente tanto el entorno social como la valoración que ante la enfermedad predomine en la cultura dominante.

María Angeles Durán habla de una doble distinción que debería hacerse a la hora de considerar la enfermedad. Una, aquellos procesos que se producen en el interior del ser humano y que son originados por los caracteres inherentes a la persona; la otra, aquellas enfermedades que advienen al individuo y que son totalmente aleatorias en su aparición.

Tal vez teniendo en cuenta esta distinción pueda entenderse el rechazo social sufrido por los dementes a lo largo de la historia. Este enfoque se justificaría buscando una relación causal entre comporta- 
miento y aparición de la locura o la enfermedad. Por ello, el aislamiento y el rechazo no suponen más que el castigo social hacia el estigmatizado.

Ya en los primeros escritos que hablan del hombre y sus enfermedades, se hallan referencias que vinculan a los desviados mentales con otras patologías de carácter infeccioso, cuyo tratamiento pasaba por el alejamiento de los enfermos de los centros urbanos, como medida de prevención.

Así la historia habla de los primeros sanatorios para apestados y leprosos, donde convivían toda clase de enfermedades, siempre que éstas fueran objeto de rechazo social.

Hay que esperar a que estas ideas evolucionen y cambien para que se distinga entre enfermedades del cuerpo y las enfermedades del alma y se separe a las víctimas de la lepra y otras infecciones de los dueños de la sinrazón.

El concepto de la demencia como enfermedad curable no tuvo repercusión alguna hasta 1770 . En ese año, según Michel Foucault, se comenzaron a aplicar en la Bicetre los tratamientos que ya en el Hotel Dieu se venían desarrollando, con poca repercusión, desde bastante tiempo atrás.

Hasta este momento, la posible curación de los alienados había sido considerada como algo totalmente imposible. Sin dejar de considerar aquellas posturas que negaban cualquier intervención de carácter rehabilitador, justificando el hecho como castigo divino para los afectados.

Paralelamente, la historia de los deficientes mentales ha sufrido también las consecuencias de estas actitudes que favorecían la separación y aislamiento.

Las referencias a débiles mentales o imbéciles datan de 1721, año en que se asociaba la imbecilidad a las tres principales enfermedades del espíritu: frenesí, manía y melancolía, tal como algunos autores las definían. La imbecilidad, según se decía entonces, podía estar producida bien por el sufrimiento prolongado de las demás, o por la sequedad del cerebro, o por golpes y caídas, o por consumo exagerado de líquidos espirituosos, o masturbación, o como consecuencia de la apoplejía. Posteriormente la terminología hablaba entonces de idiotez, cretinismo, imbecilidad, flojedad o debilidad mental.

En 1778 se afirmaba que incluso los niños con la más extrema estupidez mental podían tratarse de modo que tuvieran una existencia digna de cualquier ser humano. Fue en 1790 cuando se consiguieron notables progresos en lo cognitivo y lo social en un niño calificado como «idiota incurable y menos inteligente que un animal doméstico». Esta labor no tuvo continuidad, pero influyó notablemente en los esfuerzos de Edonard. En 1846, siendo director de una escuela para idiotas en París, publicó el primer tratado de educación de defi- 
cientes mentales, que fue la base intelectual de la obra de María Montessori.

Los adelantos más destacados se producen en Alemania, donde fueron creadas las primeras escuelas de ayuda específica para disminuidos, que datan de 1818, año en que, en Salzburgo, se funda la primera Institución Educativa para Cretinos.

En los años inmediatamente anteriores a la revolución industrial, y en ambientes principalmente agrícolas, los disminuidos llamaban poco la atención. No eran considerados un problema, al colaborar en las labores del campo y ser una mano de obra poco exigente y de elevada permanencia.

En la creación de las primeras instituciones de ayuda y formación tuvo un importante papel el factor de utilidad de los deficientes. Mediante su educación y adiestramiento el objetivo principal era conseguir convertirlos gradualmente en miembros útiles para la sociedad. Con este criterio, la selección para acceder a cualquier centro específico pasaba por la valoración de las posibilidades futuras del candidato. Los casos desestimados eran recluidos dentro de algún hospital general, con pocas posibilidades de promoción de sus facultades, o bien permanecían en el núcleo familiar, ajenos a cualquier tratamiento rehabilitador.

A partir de 1938, se crearon las primeras asociaciones de padres con hijos deficientes. Estas han ido posteriormente proliferando y junto con la iniciativa privada y la beneficencia, han sido los principales impulsores de la asistencia a los disminuidos. Sin embargo, el ancestral criterio de aislamiento todavía permanece.

Desde las primeras épocas hasta hoy, el mundo de las enfermedades de la mente parece seguir envuelto en una especie de vergonzoso hermetismo y se siguen aplicando criterios que poco dicen de la voluntad integradora de la sociedad. Según Foucault, el entorno social no llegará nunca a aceptar a aquellos de cuya enfermedad, de alguna u otra manera, se les considera culpables.

Ya se ha visto que el internamiento de aquellas personas con problemas de disminución psíquicas y físicas, como medio de aislamiento, ha sido una constante en las relaciones de estas personas con la sociedad. La estructura social no acepta a aquellos que por su especial condición se ven incapacitados para tener una vida y un desarrollo normales dentro de la misma. La plasmación más evidente de este rechazo fue la creación de centros para su internamiento.

La sociedad ha demostrado a través de la historia que está incapacitada para acoger de otra manera a los considerados miembros no productivos. En el caso concreto de los problemas psiquiátricos, crea instituciones donde se les mantenga, se atiendan sus necesidades vitales y sobre todo donde permanezcan relegados en un plano que no por asumido puede considerarse el más justo. 
Actualmente las instituciones dedicadas a la ayuda y atención de disminuidos psíquicos, en general, siguen el siguiente esquema:

- Centros de estimulación precoz. Centros de educación preescolar.

- Centros reeducativos. Colegios de integración.

- Centros ocupacionales.

- Residencias e internados.

Los centros de estimulación precoz suponen el primer eslabón en la cadena. A ellos acuden, casi siempre en régimen ambulatorio, tanto deficientes que hayan sido reconocidos médicamente como tales e independientemente de su grado de deficiencia, como otras diversas patologías, que si bien no están relacionadas con el retraso mental, precisan ayuda para su correcto desarrollo psicomotriz.

En los centros de educación preescolar se atiende aquellos casos en edad escolar susceptibles de formación y educación. Son centros educativos especializados y que están muy estrechamente relacionados con los anteriores. Suponen la alternativa más normalizada dentro del aspecto educativo, ya que difieren poco en sus planteamientos y finalidades de los centros de educación usuales.

Los colegios de integración son una posibilidad más dentro del panorama educativo. En ellos, y dependiendo de la modalidad elegida, que puede ser aula de apoyo o bien ayuda suplementaria, se trata de aprovechar la edad escolar del deficiente para el aprendizaje.

Los centros ocupacionales recogen una minoría muy selectiva de disminuidos que pueden ser instruidos en la realización de sencillos trabajos. El fin hipotético de estos centros sería la integración laboral del individuo, aunque siempre contando y/o dependiendo del mayor o menor grado de incapacitación con que se cuente.

Por último estas residencias e internados que son la alternativa que nos ocupa. Son centros que acogen aquellos casos que parecen imposibles de mantener dentro del entorno familiar y social. No siempre el interno carece de familiares allegados que puden responsabilizarse de él y no siempre su estado de deficiencia hace imposible que permanezca dentro de su localidad y su familia.

Existen gran cantidad de factores que hacen erigirse a este tipo de centros como la única posibilidad para un problema: el de tener un deficiente mental en casa.

Posiblemente existen algunos casos que están plenamente justificados. Son casos como los de inexistencia de familiares allegados o directos o de excesiva agresividad o de total dependencia del enfermo u otros casos extremos. Pero no son pocos los casos en que un miembro de la familia es internado porque las características distintivas de éste hacen que resulte una carga demasiado pesada. De este modo el centro/residencia es en algunos casos la solución ideal. El 
internamiento refleja, en muchas ocasiones, la no aceptación de los enfermos. En algunas ocasiones, hay un sentimiento de culpabilidad hacia él; otras veces, el rechazo viene motivado por la desestabilización del núcleo familiar, aunque el deficiente no sea responsable directo de éste.

\section{DELIMITACION GEOGRAFICA DE NUESTRO ESTUDIO}

El trabajo de campo que hemos realizado lo situamos en un centro situado en la provincia de Alicante. Es un centro dedicado a la atención de los minusválidos psíquicos profundos y adultos, donde se les procura una asistencia adecuada, así como la posibilidad de su desarrollo personal y de una posible integración social.

La atención que el centro presta al minusválido, se efectúa en régimen de residencia. Los objetivos principales del centro son:

a) Acoger a aquellos disminuidos que por sus características personales y sociofamiliares necesiten una residencia.

b) Crear un ambiente de acogida de forma que los residentes sientan que el centro es un hogar para ellos.

c) Dar una atención integral a los disminuidos que dé respuesta a sus necesidades específicas.

d) Potenciar la relación con la familia y con el entorno social.

Los fines del centro se concretan en responder a las necesidades humanas y personales del residente. Intentan que tenga un carácter alegre y procuran evitar darle un aspecto hospitalario. Consideran que debe ser un hogar, con una faceta asistencial y sanitaria.

El centro apuesta también por acciones complementarias como son:

a) Favorecer la formación del personal.

b) Potenciar las acciones encaminadas a modificar el entorno social con el fin de favorecer una mayor integración del deficiente.

c) Ser un lugar dedicado a la asistencia, atención y mantenimiento, así como tratamientos idóneos continuados para lograr, a ser posible, su integración en la sociedad.

El tipo de población interna que tiene el centro es la siguiente: 


\section{TABLA DE EDADES}

Población

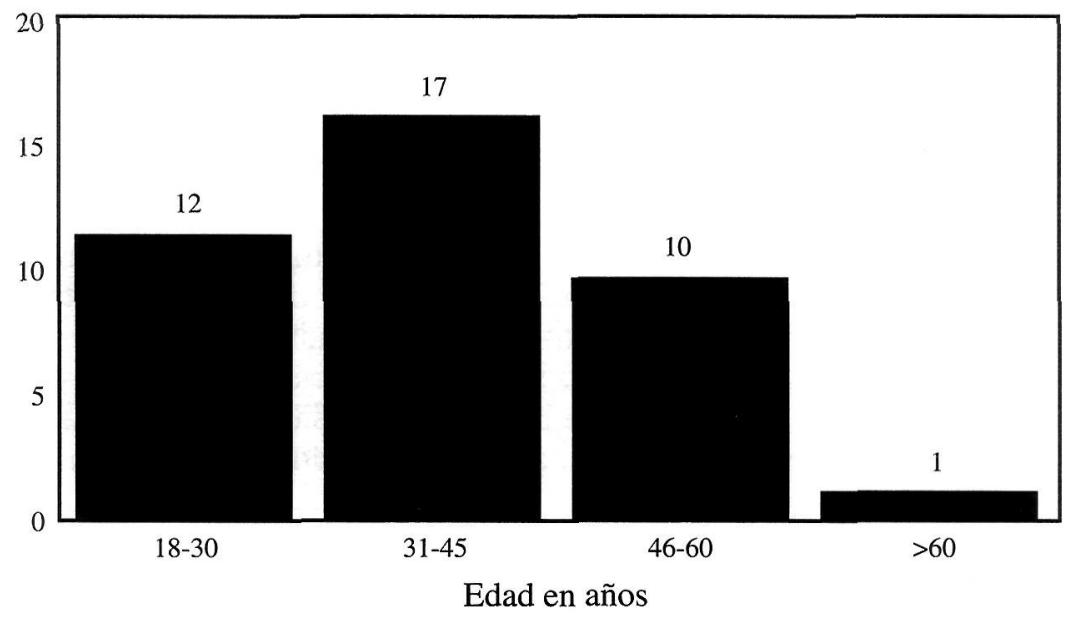

TIPO DE ORFANDAD

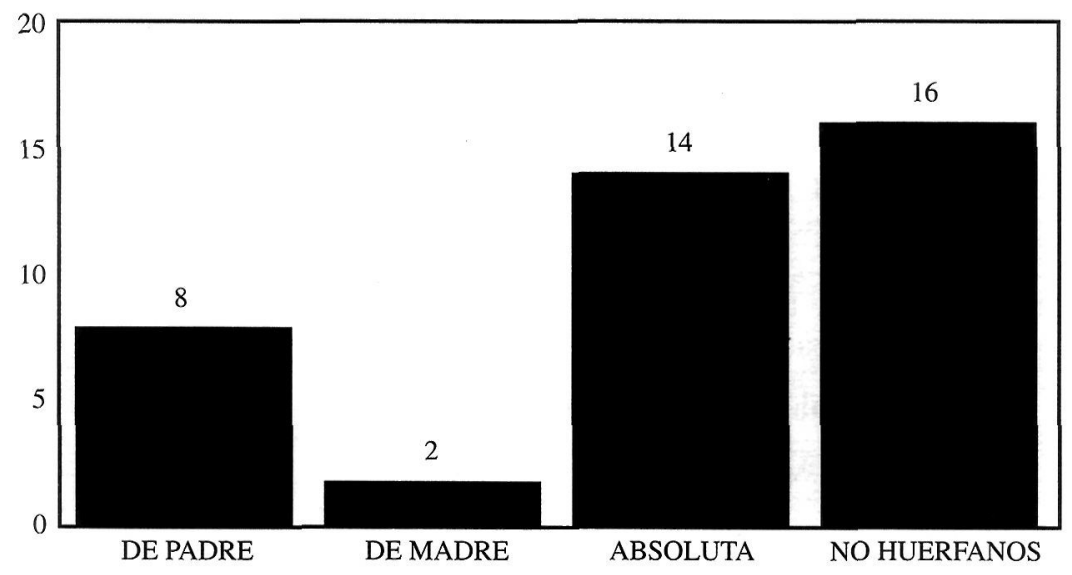




\section{GRADO DE DEFICIENCIA}

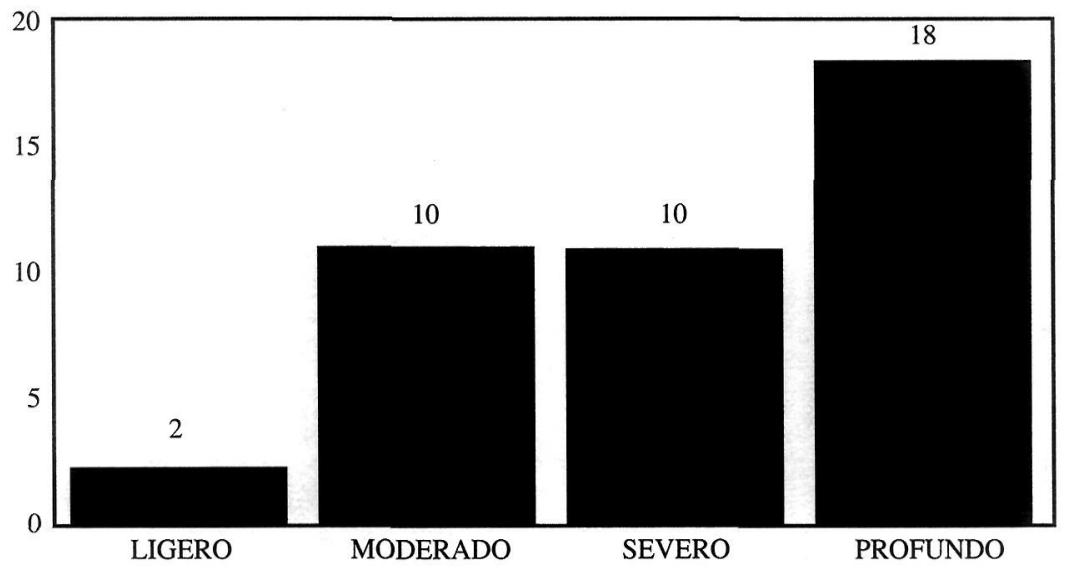

ETIOLOGIA

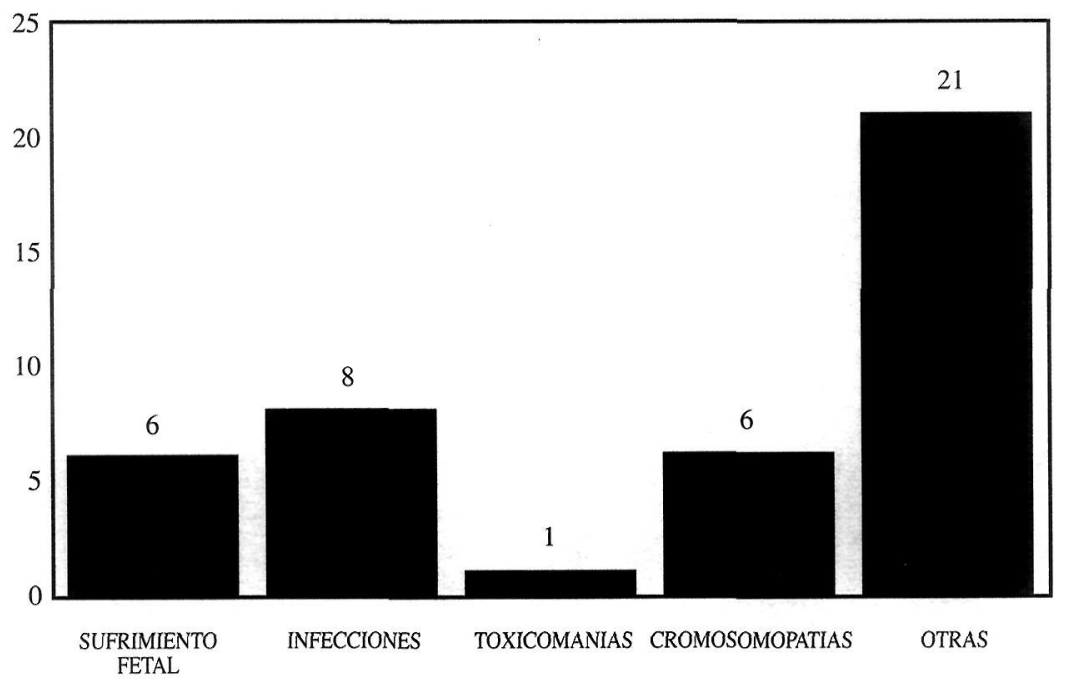




\section{CONDUCTAS AGRESIVAS}

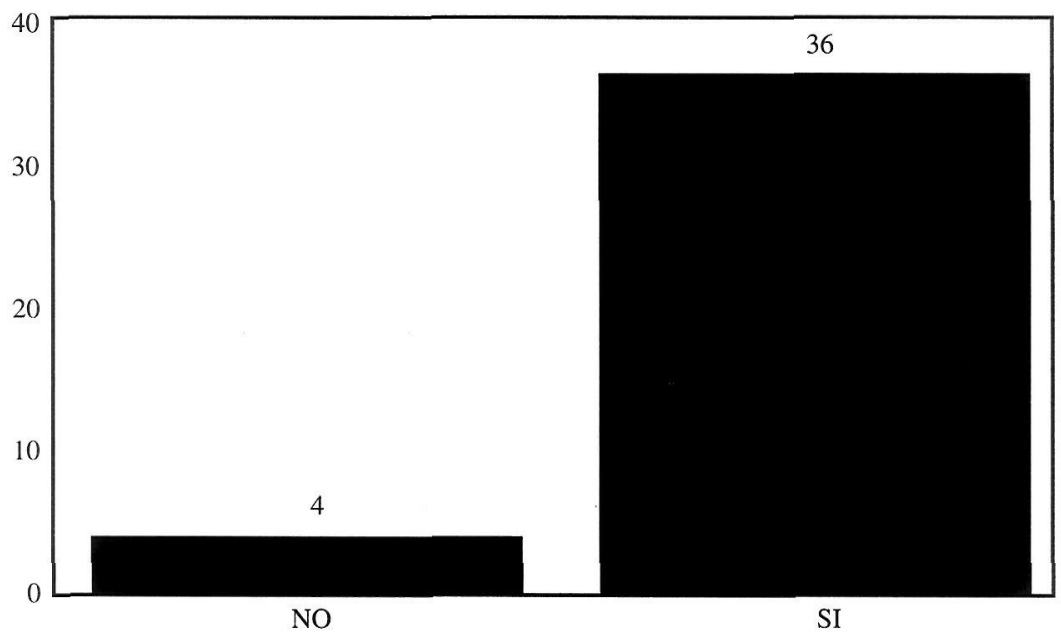

EPILEPSIA

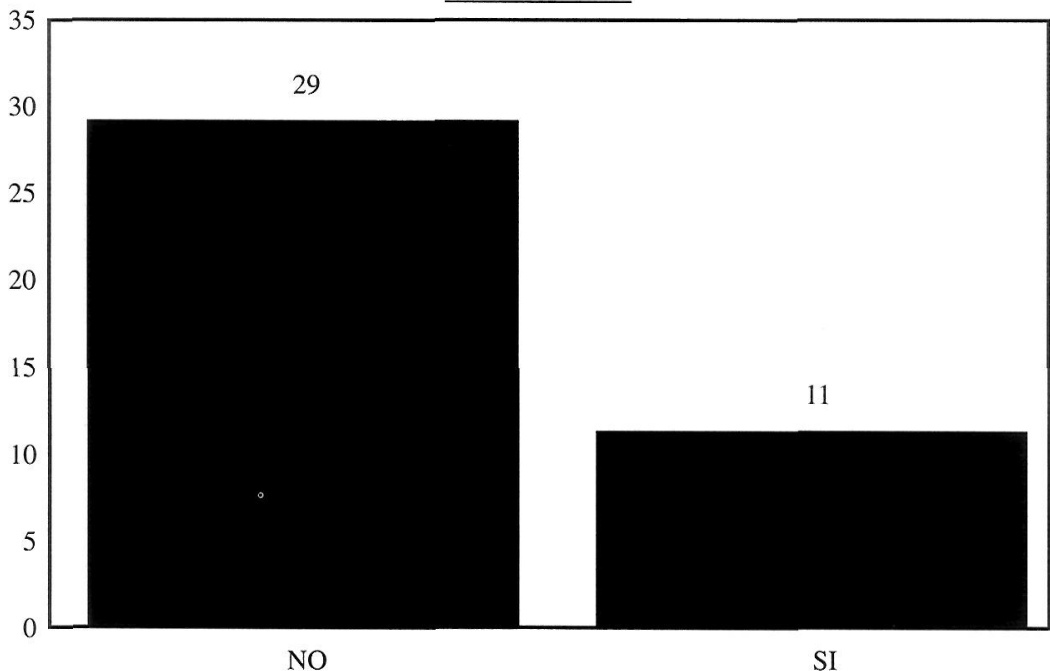




\section{LENGUAJE}

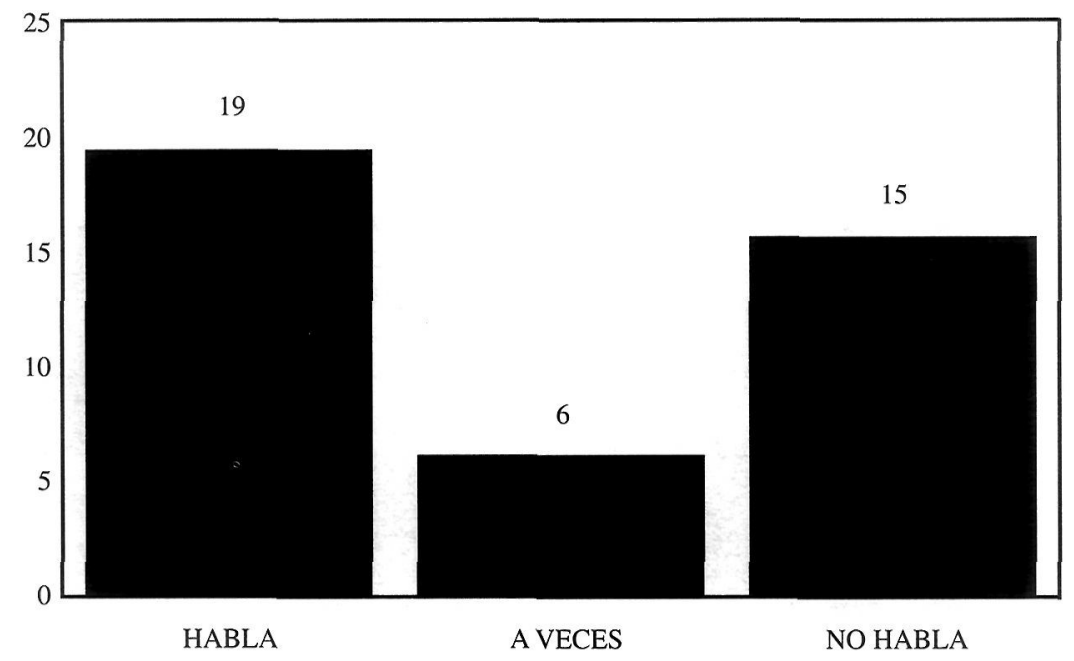

CONTROL DE ESFINTERES

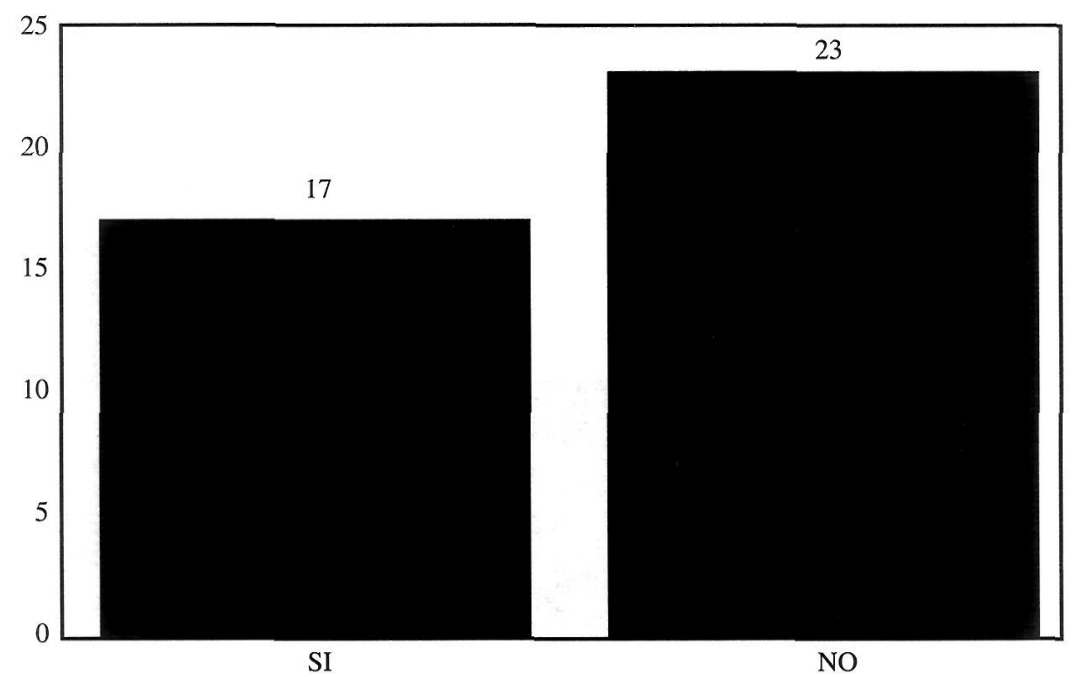


HABITOS DE MESA

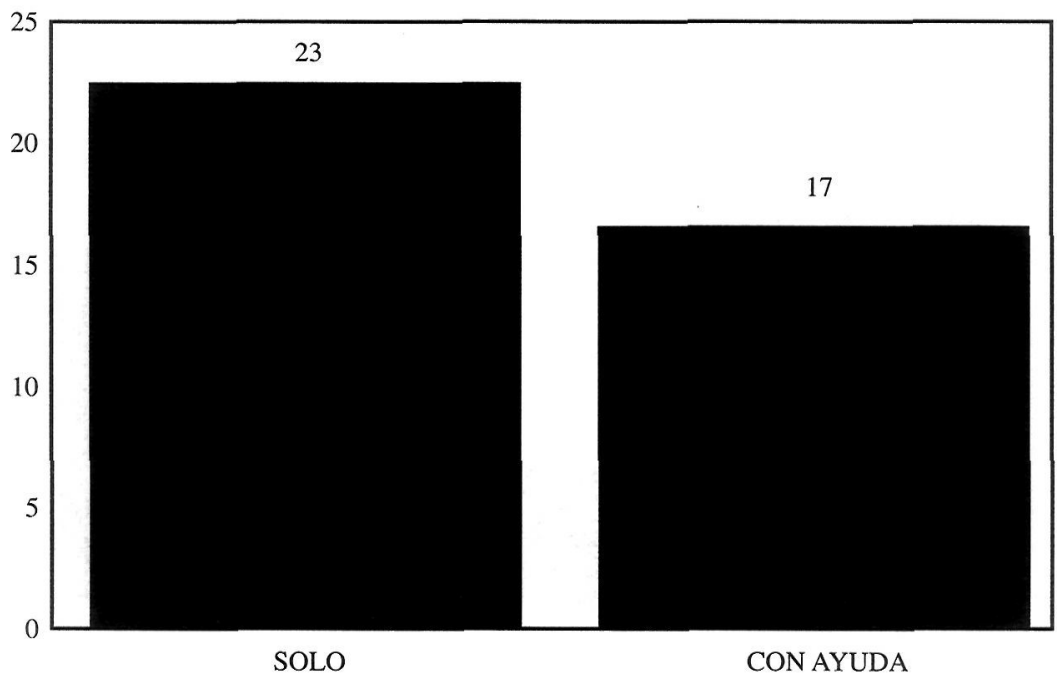

\section{LOCOMOCION}

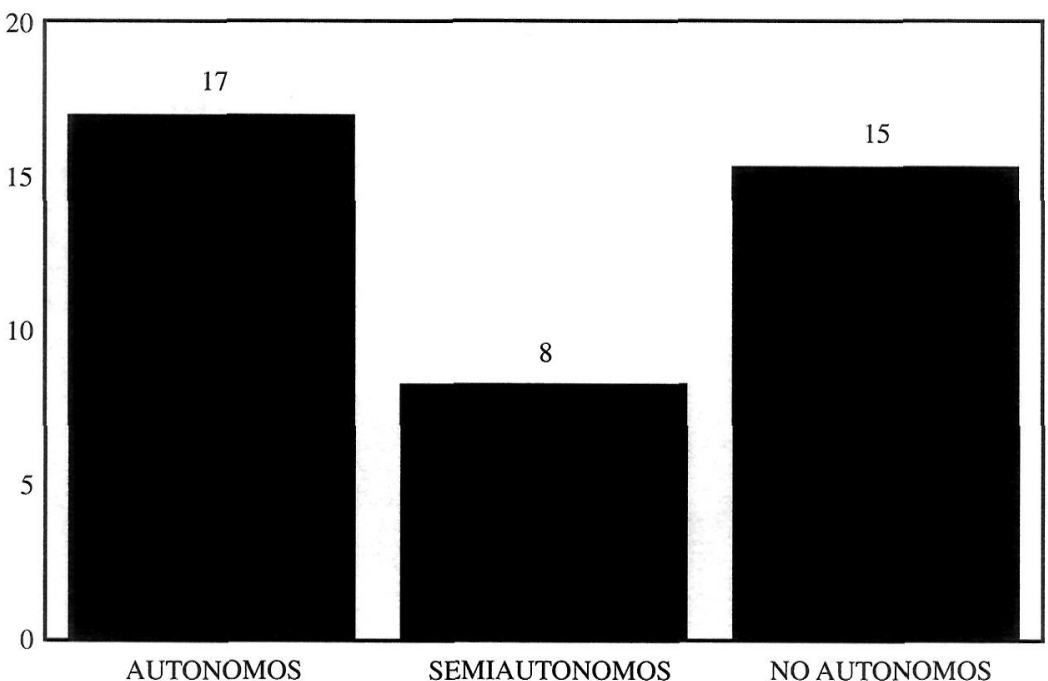




\section{VISION}

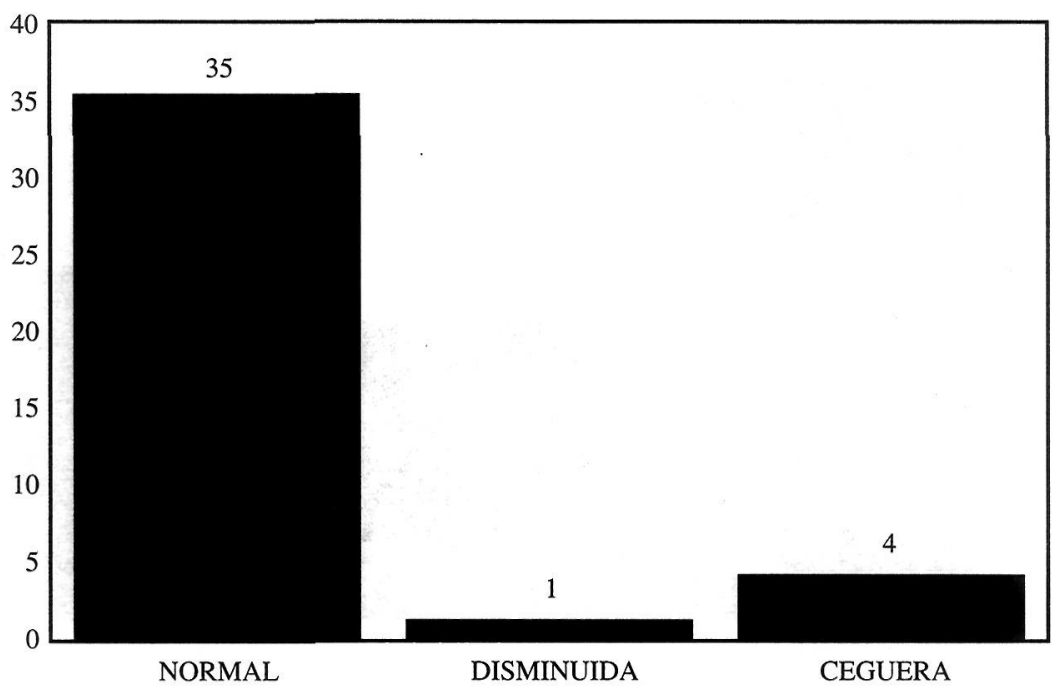

\section{AUDICION}

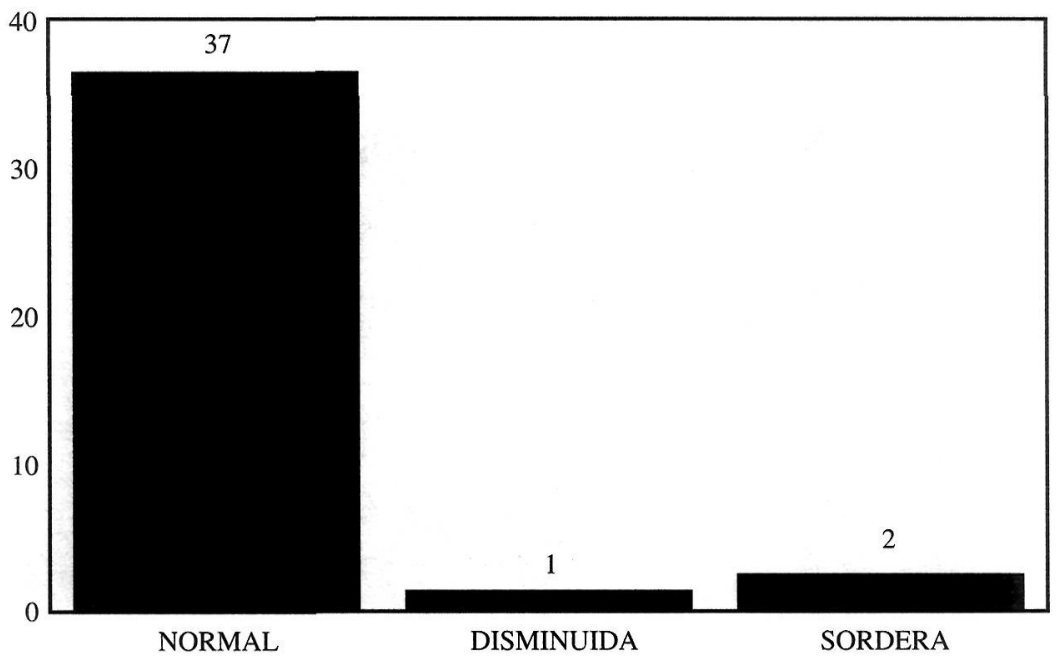




\section{HABITOS DE VESTIDO}

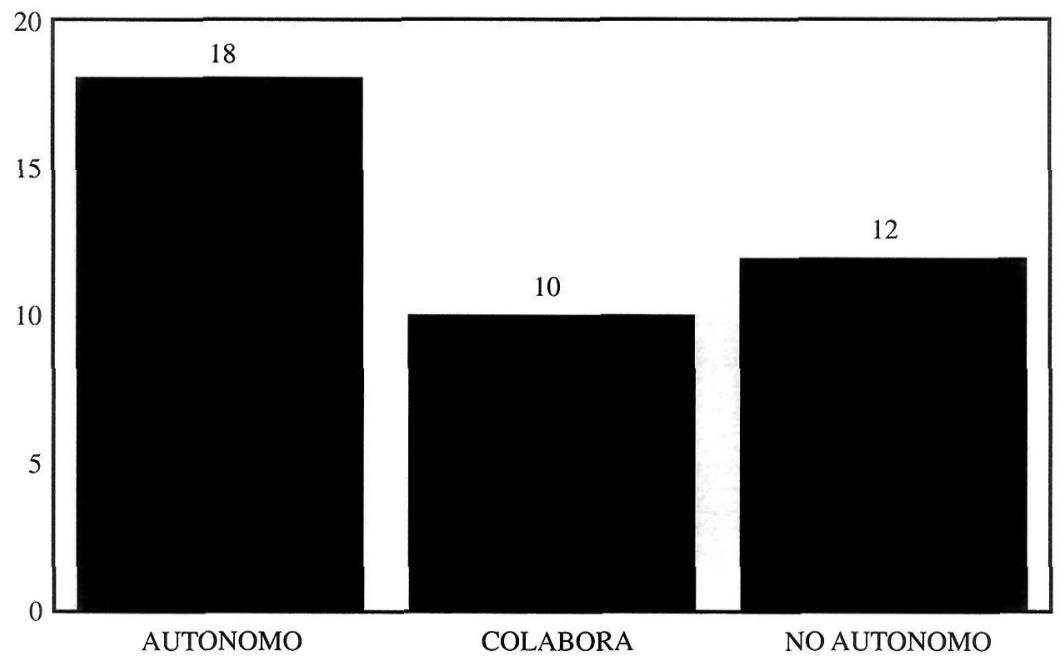

HABITOS DE ASEO

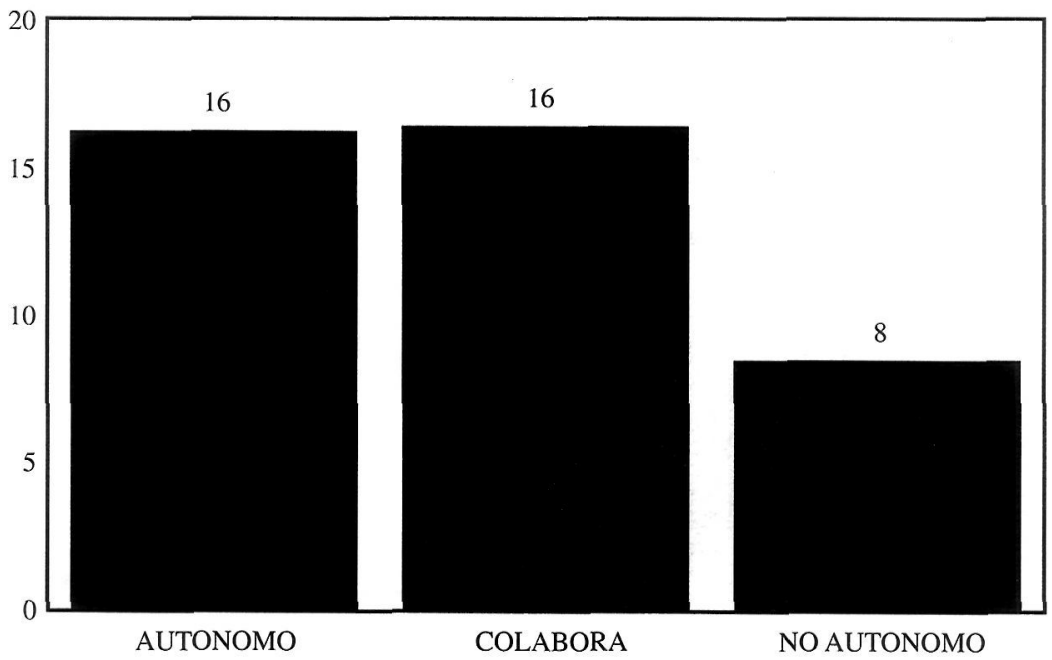


De los informes realizados en el momento del ingreso se puede deducir que existe una desestructuración familiar característica en casi todas de ellos. Son muy pocos los internos que proceden de un entorno familiar normalizado y los condicionantes de inestabillidad o precariedad económica son evidentes en la mayoría de ellos. Al menos siete de las familias que tienen un interno en el centro, cuentan con otro deficiente entre sus miembros.

Los contactos de los internos con sus familias son poco frecuentes. En algunos casos son incluso inexistentes desde hace años. Solamente 12 de los 40 internos que cuenta el centro, reciben visitas regulares y aportan la ropa necesaria ante cada cambio estacional. El centro ha asumido la total responsabilidad y tutela de los internos y les provee de todo lo necesario ante el desentendimiento de sus familias.

Igualmente difícil se plantea el análisis de las causas del internamiento. Indudablemente, hay casos en los que la avanzada edad de los padres y el desinterés por parte de los familiares cercanos, es la motivación más comprensible, junto con la pérdida de los padres, de los hermanos o del miembro de la familia que se ocupaba con anterioridad del deficiente.

De la población de este centro, solamente 7 de los internos podrían llevar una vida normalizada sin que su presencia fuera un problema demasiado grave en el contexto de un entorno familiar. Pero muchos de ellos carecen de este entorno, por lo que su internamiento en el centro es la única posibilidad con que se encuentran, dejando aparte si el centro por sus características de residencia para deficientes profundos adultos, puede ser o no el ambiente adecuado para ellos.

El resto de los residentes merecen otro tipo de atención y dedicación que pocas veces encontrarían residiendo en sus domicilios respectivos. Los deficientes mentales profundos necesitan una atención y cuidado que difícilmente se pueden encontrar en otras personas que no sean sus propios padres.

$\mathrm{Al}$ tratar de establecer las posibles razones del internamiento de las personas que componen la población del centro, aparecen tres grupos principales:

1. Aquellos que no podrían verse integrados en una familia normal, por necesitar una constante atención.

2. Aquellos que han sido ingresados porque al faltar sus padres nadie ha asumido la responsabilidad de su cuidado.

3. Aquellos que por sus condiciones especiales, han sido considerados un lastre para el normal funcionamiento de su entorno familiar y han sido apartados y relegados a la indiferencia e incluso al olvido. 
La jornada de un día normal en el centro, comienza a las 8 de la mañana, cuando el turno de cuidadores de la mañana llega al centro reemplazando a los cuidadores que realizan el turno de noche. Algunos de los residentes ya han sido levantados, duchados y vestidos, atendiendo a las necesidades de cada uno. Así, hay un grupo de 6 internos que se levantan a las 7 de la mañana, con el fin de estar preparados a las 8 para trasladarse a un centro ocupacional. Este grupo quizás pueda considerarse una selección de entre los demás, puesto que por sus especiales características, han sido considerados susceptibles de formación, y con ese fin acuden todos los días al centro ocupacional hasta las 5 de la tarde. El resto de residentes aguardan al cuidador en su habitación para que él reparta la ropa del día, duche y afeite a los que lo necesitan.

Hacia las 9 de la mañana, una vez realizadas las tareas de aseo y limpieza, pasan al comedor para que sea servido el desayuno. Posteriormente, existe un espacio de tiempo, hasta las dos del mediodía, en que las actividades son variadas. El grupo al que le corresponda, por turno, podrá salir de paseo. Los que permanecen en el centro, bien realizan los denominados «talleres», en los que se dedican a tareas sencillas como montajes de cubos, construcción de puzles, ensamblado de bolas, etc., o se ocupan en la ejecución de algún tipo de gimnasia rehabilitadora con la terapeuta ocupacional o con la psicóloga del centro.

Esta distribución del tiempo se mantiene hasta la 1 del mediodía, hora en la que habitualmente se sirve la comida, que es distribuida en 2 turnos. En el primero comen los considerados profundos, esto es que necesitan ayuda total o parcial para comer, acudiendo al segundo aquellos otros cuyo comportamiento en la mesa es autónomo.

Terminada la comida van pasando por los aseos, donde se lavan los dientes, la cara y las manos. Se debe resaltar el control de esfínteres que los profundos realizan cada 2 o 3 horas, según el grado de contención de cada uno y que se realizan con el horario citado durante toda la jornada, incluido el horario nocturno. Una vez realizado el control correspondiente, todos los internos permanecen en la sala de estar esperando a los cuidadores del turno de la tarde y la hora de la merienda. El grupo de autónomos, junto con el grupo de medios, salen a pasear o bien se desplazan hacia las aulas para seguir con los talleres de manualidades.

Hacia las 7,30 de la tarde se reciben las cubetas que traen la ropa limpia de los lavaderos y que es distribuida por las habitaciones; cada cuidador prepara la que vestirán los internos al día siguiente. La posibilidad de que puedan elegir su propia ropa queda supeditada a las necesidades de la lavandería.

Aproximadamente a las 8 se vuelve a repetir el mismo proceso de la hora de la comida para la cena. Tras la cena todos los internos pa- 
san por el lavabo para lavarse o ducharse aquellos que por la mañana no lo han hecho y los que por su especial sudoración lo requieran. Este aseo e higiene suele durar hasta las 9,30, hora en que son acostados en sus camas respectivas y termina para ellos la jornada.

Algunos internos prefieren quedarse viendo la televisión hasta la llegada de los cuidadores del turno de noche, aunque no muestran un especial interés por la programación, puesto que una vez realizado el cambio de turno todos se acuestan.

Cabría distinguir 2 clases de relaciones entre los residentes:

1. Relaciones entre los internos.

2. Relaciones de los internos con el personal laboral.

Las relaciones entre los internos son muy escasas. Pudiera decirse que han aprendido a convivir unos con otros sin molestarse ni interferirse. Amistad y camaradería son prácticamente inexistentes, a pesar de estar conviviendo durante tanto tiempo. Sin llegar a la rivalidad, cada uno delimita perfectamente su espacio, permitiendo muy pocas veces que un compañero lo invada. Este comportamiento aparece mucho más destacado entre los autónomos, ya que a propósito de los profundos hablar de cualquier reflexión al respecto sería muy aventurado.

Dentro del grupo de autónomos, las relaciones que pueden darse son con los que acuden diariamente al centro ocupacional. Y este hecho propicia que el contacto entre ellos sea más acusado que entre los demás. Aún así, parecen mantener las distancias, sin dejar que ninguno interfiera en su mundo particular. Quizás podríamos decir que la situación del internado propicia el individualismo y que ninguno deja de considerar al compañero como un extraño. En los denominados profundos, es muy probable que ni siquiera exista el concepto del YO diferenciado. Con ello la percepción de otros es bastante improbable. Existen relaciones esporádicas entre ellos, pero casi siempre a instancias del cuidador.

Las relaciones personales entre los internos están prácticamente supeditadas a sus relaciones con los cuidadores, esto es, los residentes demandan continuamente la atención del cuidador, sin tener la necesidad de establecer ningún otro tipo de relación.

La demanda de atención de los enfermos al encargado de su habitación es constante; mientras éste provea todas sus necesidades, cualquier otra cosa es secundaria.

Podría decirse tal vez que la labor del cuidador debería pasar por evitar esta dependencia; pero hay que considerar también otro factor importante: y es que la mayoría de los residentes del centro, debido en parte a la deficiencia que padecen, o bien por no haber recibido una adecuada estimulación durante la infancia, sufren un bloqueo emocional que les impide, a modo de barrera, establecer contacto con el exterior. Las dificultades comunicativas son, en algunos casos, ex- 
tremas y no existe la más mínima intención por parte del interno de comunicarse. Incluso con el trato diario y la satisfacción de sus necesidades, resulta casi imposible saber cuál es su estado de ánimo, dado que la expresión manifiesta es inexistente. A este comportamiento pueden buscarse todo tipo de explicaciones o causas: desde el rechazo sufrido durante largos períodos de su vida, hasta la incapacidad sensorial para la comunicación.

Las relaciones de los internos con el medio ajeno al centro están supeditadas a sus salidas al exterior. Diariamente realizan salidas aquellos que acuden al centro ocupacional, con lo que en este grupo el grado de aislamiento es ligeramente inferior, dado que esta circunstancia les permite relacionarse con personas ajenas al centro.

En cuanto a los que permanecen en el interior del centro, el contacto queda limitado a los días en que, por turno, les corresponda salir. Solamente un número pequeño de internos reciben visitas y pueden salir con sus familiares y estar, aunque por espacios reducidos de tiempo, totalmente fuera de lo que podíamos considerar el ambiente de la residencia. Son los mismos que en períodos vacacionales pueden volver a sus hogares.

La noción de pertenencia a un medio distinto al del propio centro solamente es evidente en 3 de los internos, aunque no sean éstos precisamente los que cuentan con mayor atención por parte de sus familias.

Durante las salidas lo más normal en su actitud es la extrañeza, ya sea porque ésa es la actitud que perciben en la gente con la que se encuentran, ya sea porque, para ellos, cualquier salida es una novedad dentro de la monotomía diaria.

También cuentan con la posibilidad de realizar un campamento durante los meses de verano. Ante la proximidad de esas fechas resulta difícil contener sus emociones, ya que para ellos dejar la residencia durante tanto tiempo supone un gran acontecimiento.

La residencia, por su carácter de institución total, comprende la gran mayoría de las características que definen a este tipo de instituciones. Gran parte de éstas han sido definidas y estudiadas por Goffman en su ensayo sobre la situación social de los enfermos mentales (Goffman, 1970: 18, 19). En términos generales nuestro estudio, puede situarse dentro de los límites marcados por Goffman.

\section{LOS ENFERMOS MENTALES Y LOS CONDICIONAMIEN- TOS INSTITUCIONALES}

En la obra de Goffman, se exponen algunos criterios que consideramos orientadores a la hora de afrontar el problema específico de nuestro estudio. Entre ellos destacan los que se refieren, por ejemplo, a: 
- La percepción del yo. Resulta difícil en un ambiente en donde la mayoría de los individuos tiene un retraso intelectual de al menos un $15 \%$, admitir que su personalidad sea dañada en la mínima expresión del concepto. Aparte del retraso y de las disfunciones, tanto mentales como físico-motrices, hay que considerar que en el momento de su llegada al centro, procedían de variados lugares donde las percepciones del yo de cada uno habían sido notablemente dañadas. Es prácticamente imposible asegurar con un mínimo de certeza que lo más íntimo de la personalidad de cada uno haya permanecido intacto hasta su ingreso. No debemos olvidar que se trata de un centro para adultos.

- La contaminación física: El contacto personal continuado, tanto con la población de internos como con el colectivo de trabajadores, tiene efectos importantes en cuanto a la asunción de un rol de sometimiento. Puede considerarse que son 3 los momentos a lo largo del desarrollo de la vida diaria, en que hay contacto entre los internos: dormitorios comunes, uso de los servicios de higiene y duchas colectivas. Al igual que el estudio de Goffman, estas 3 situaciones son de gran importancia como factores que favorecen la desindividualización de los enfermos y de algún modo, la pérdida de su identidad personal.

La violación de la intimidad es un hecho que expone claramente a los internos a aceptar un tipo de relación con los demás. De igual manera este sometimiento se produce respecto al personal laboral del centro.

Las relaciones paciente-cuidador terminan por ser impositivas cuando de cada hecho cotidiano contenido dentro de los objetivos/fines del centro termina desprendiéndose un criterio rígido de comportamiento; una pauta de conducta inflexible e inmodificable. Así, tanto en el inicio de la jornada por la mañana como el desarrollo del día, están repletos de situaciones en las que el interno cede continuamente parte de su albedrío a manos del cuidador que preserva la costumbre y hábitos establecidos. Esta situación es la que Goffman denomina: «elaboración del mundo a través de privilegios». De esta forma, el verdadero sentido de la institución es: residencia «para» el cuidado y tratamiento y control de disminuidos.

Esto parece corroborar el resultado de los primeros estudios de comunidad en un hospital psiquiátrico, tras los cuales se llegó a la conclusión de que el principal propósito de la institución era el control de los enfermos sin tener en cuenta su bienestar.

Levison y Galleguer (1964) hacen una réplica o crítica a lo que consideran conceptos totalitarios de Goffman, respecto al efecto nocivo que sobre la identidad del individuo tienen las instituciones. 
Pensamos que tal planteamiento queda en este caso fuera de lógica, dado que la capacidad de respuesta, y menos de rebelión, del interno hacia una permanencia menos condicionada, es muy improbable.

\section{REFLEXIONES FINALES}

Al término de nuestra exposición, entendemos que es obligado aludir al hecho de que el mundo de los deficientes mentales es un universo complejo teñido de diversos matices, pero ante todo debe tenerse presente la consideración de que estamos tratando siempre con seres humanos y por ello cualquier intento de agrupación y clasificación resulta a todas luces aventurado.

Las características distintivas de cada persona parecen duplicarse, incluso multiplicarse, cuando se habla de disminuidos psíquicos, porque cada uno conlleva un pasado, casi siempre desconocido, y un historial que puede ser determinante a la hora de establecer nuestra relación con ellos.

Al tratar de analizar el desarrollo de la vida en una residencia para profundos y con una población que lleva tras de sí, al menos, 9 años de internado y de separación del entorno social, resulta difícil discernir cuáles de sus conductas han sido impuestas por la dinámica del centro y cuáles de ellas son herencia de un pasado anterior. En suma, el análisis de la vida dentro de estos centros no puede hacerse más que teniendo presentes las normas, hábitos y actividades que han sido de alguna manera interiorizadas por los internos y que son indispensables para el correcto funcionamiento de su vida interior y para hacer posible que se cumplan los fines específicos de la institución.

\section{BIBLIOGRAFIA}

Comelles, J. M.: «Ve no sé dónde, trae no sé qué. Algunas reflexiones sobre el trabajo de campo en Antropología de la Salud». En Arxiu d'Etnografía de Catalunya, 7.205-235, Tarragona, 1989.

Durán, M. A.: Desigualdad social y enfermedad. Ed. Tecnos, Madrid, 1983.

Foucault, M.: Historia de la locura en la época clásica. Fondo de Cultura Económica, tomos I y II, México, 1985.

-: Enfermedad mental y personalidad. Ed. Paidos Studio, México, 1988.

Goffman, E.: Internados. Ensayos sobre la situación social de los enfermos mentales. Ed. Amorrortu, Buenos, Aires, 1963.

Kenny, M. y Miguel, J. M.: La antropología médica en España. Ed. Anagrama, Barcelona, 1980.

Levinson, D. J. y Gallagher, E. B.: Sociología del enfermo mental. Ed. Amorrortu, Buenos Aires, 1988. 